\title{
Pachydrusen: the epidemiology of pachydrusen and its relevance to progression of pachychoroid disease spectrum
}

\author{
Jay Sheth ${ }^{1}$ - Giridhar Anantharaman ${ }^{1} \cdot$ Nilesh Kumar $\mathbb{D}^{2} \cdot$ Nikulaa Parachuri $^{2} \cdot$ Francesco Bandello $^{3}$. \\ Baruch D. Kuppermann ${ }^{4}$. Anat Loewenstein ${ }^{5}$. Ashish Sharma ${ }^{2}$
}

Received: 21 March 2020 / Revised: 8 April 2020 / Accepted: 8 April 2020 / Published online: 23 April 2020

(c) The Royal College of Ophthalmologists 2020

Pachydrusen (PD) has recently been described as a unique form of drusen associated with increased choroidal thickness. They are yellowish-white sub-RPE deposits having a wider distribution at the posterior pole. They have welldefined margins with more complex irregular outer contour and occur in isolation or in groups of few [1]. Spaide et al. have described it in patients with non-exudative age-related macular degeneration (AMD) while Lee et al. have described it in patient with polypoidal choroidal vasculopathy (PCV) [2]. Numerous studies have shown the presence of drusen to be an independent risk factor for progression to advanced AMD, including geographic atrophy (GA) and choroidal neovascularization (CNVM) [3, 4]. However, there is dearth of sufficient epidemiological data and no study till date has analyzed the long-term sequelae of pachydrusen in pachychoroidopathy. In the present study, we aim to report the prevalence of pachydrusen and to determine whether the presence of pachydrusen is innocuous or if it can be a potential risk factor for pachychoroid sequelae such as the development of active CSCR or type 1 CNVM \{pachychoroid neovasculopathy (PNV) / PCV \}.

$\triangle$ Ashish Sharma

drashish79@hotmail.com

1 Department of Vitreo-Retina-, Giridhar Eye Institute, Kochi, Kerala, India

2 Lotus Eye Hospital and Institute, Avinashi Road, Coimbatore, Tamil Nadu, India

3 University Vita-Salute, Scientific Institute San Raffaele, Milano, Italy

4 Gavin Herbert Eye Institute, University of California, Irvine, Irvine, CA, USA

5 Division of Ophthalmology, Tel Aviv Sourasky Medical Center and Sackler Faculty of Medicine, Tel Aviv University, Tel Aviv, Israel
We retrospectively evaluated the clinical case records and multimodal imaging of consecutive patients with pachychoroidopathy with a minimum follow-up duration of one year, who presented to the vitreoretinal services at Giridhar Eye Institute, Kochi, Kerala, India between January 2015 and April 2016. This study was conducted in accordance with the tenets of Declaration of Helsinki, and was approved by the Institutional Review Board. Written informed consent was obtained from each patient before any imaging procedures were done as part of routine clinical care. Pachychoroid characteristics on multimodal imaging were defined as the presence of any one of the following features: choroidal thickness greater than $270 \mu \mathrm{m}$ on EDIOCT and/or presence of pachyvessels [5, 6]. On the basis of multimodal imaging, the eyes were categorized into four pachychoroid variants: Pachychoroid pigment epitheliopathy (PPE), Acute /Chronic central serous chorioretinopathy (CSCR), Pachychoroid neovasculopathy (PNV), Polypoidal choroidal vasculopathy (PCV). At baseline, all patients underwent color fundus photography (CFP) (Zeiss Visupac ${ }^{\circledR}$ FF4 and FF450-plus, Carl Zeiss, Dublin, CA) and spectral-domain optical coherence tomography (SD-OCT) (Spectralis HRA + OCT, Heidelberg Engineering, Heidelberg, Germany), including enhanced depth imaging OCT (EDI-OCT). The presence or absence of PD was scrutinized on CFP for all the patients by a single masked observer (J. S.). The presence of PD was also correlated on SD-OCT. The patients were followed up with serial CFP, SD-OCT and EDI-OCT. Prevalence of PD at baseline was noted and progression to disease activity at its site was analyzed longitudinally by a single masked observer (J.S.). In the presence of disease activity (CSCR / PNV /PCV), additionally, the patients also underwent digital fluorescein angiography (DFA) and indocyanine green angiography (ICGA). Statistical analysis was done using the SPSS software, version 16.0 (SPSS Inc., Chicago, IL, USA). All data were expressed in the form of mean \pm standard deviation. Variations in age and mean follow-up duration 
between the groups were performed using the analysis of variance test, and post hoc analysis was done to look for statistical differences between individual groups. A $\mathrm{P}$-value of less than 0.05 was considered to be statistically significant.

Overall, 131 eyes of 79 patients with a diagnosis of pachychoroid were included. These included 36 eyes with PPE, 45 eyes with CSCR, and 50 eyes with PCV. The mean age of the patients was $60.2 \pm 11.22$ years. Patients with CSCR were significantly younger compared to other pachychoroid variants, namely PPE and PCV. Also, patients with PPE were significantly younger compared to PCV (PPE: $63.41 \pm 10.65$ years; CSCR: $50.53 \pm 8.55$ years; PCV: $66.58 \pm 7.28$ years; $p<0.0001)$. The mean follow-up period of the study population was $25.1 \pm 8.2$ months. Pachydrusen was observed in 70/131 eyes $(53.44 \%)$ in the pachychoroid disease spectrum. On subgroup analysis, the prevalence of PD was $41.67 \%, 60 \%$ and $56 \%$ in PPE
(15/36 eyes), CSCR (27/45 eyes), and PCV (28/50 eyes) respectively. On longitudinal follow-up of the eyes with PD, 1/15 PPE eyes (6.67\%) and 8/27 CSCR eyes (29.63\%) developed disease activity, in the form of leakage on DFA corresponding to the site of PD. Progression of PD to PCV (Aneurysmal type $1 \mathrm{CNVM}$ ) development was noted in $9 / 28$ eyes $(32.14 \%)$, that was confirmed by co-localization of CFP with multimodal imaging, including OCT, DFA and ICGA. When available, eye-tracked OCT with simultaneous DFA/ICGA was utilized for co-localizing the site of disease activity (CSCR leak or Active PCV [Polyps and/or Branching vascular Network $\{\mathrm{BVN}\}]$ ) with the locus of PD. The representative cases have been shown in Fig. 1

The primary objective of the study was to evaluate if pachydrusens are innocuous or if they can be a potential risk factor for pachychoroid sequelae. With the study results, we propose that akin to the hard and / or soft drusen of AMD, the PD are also associated with long-standing damage to the
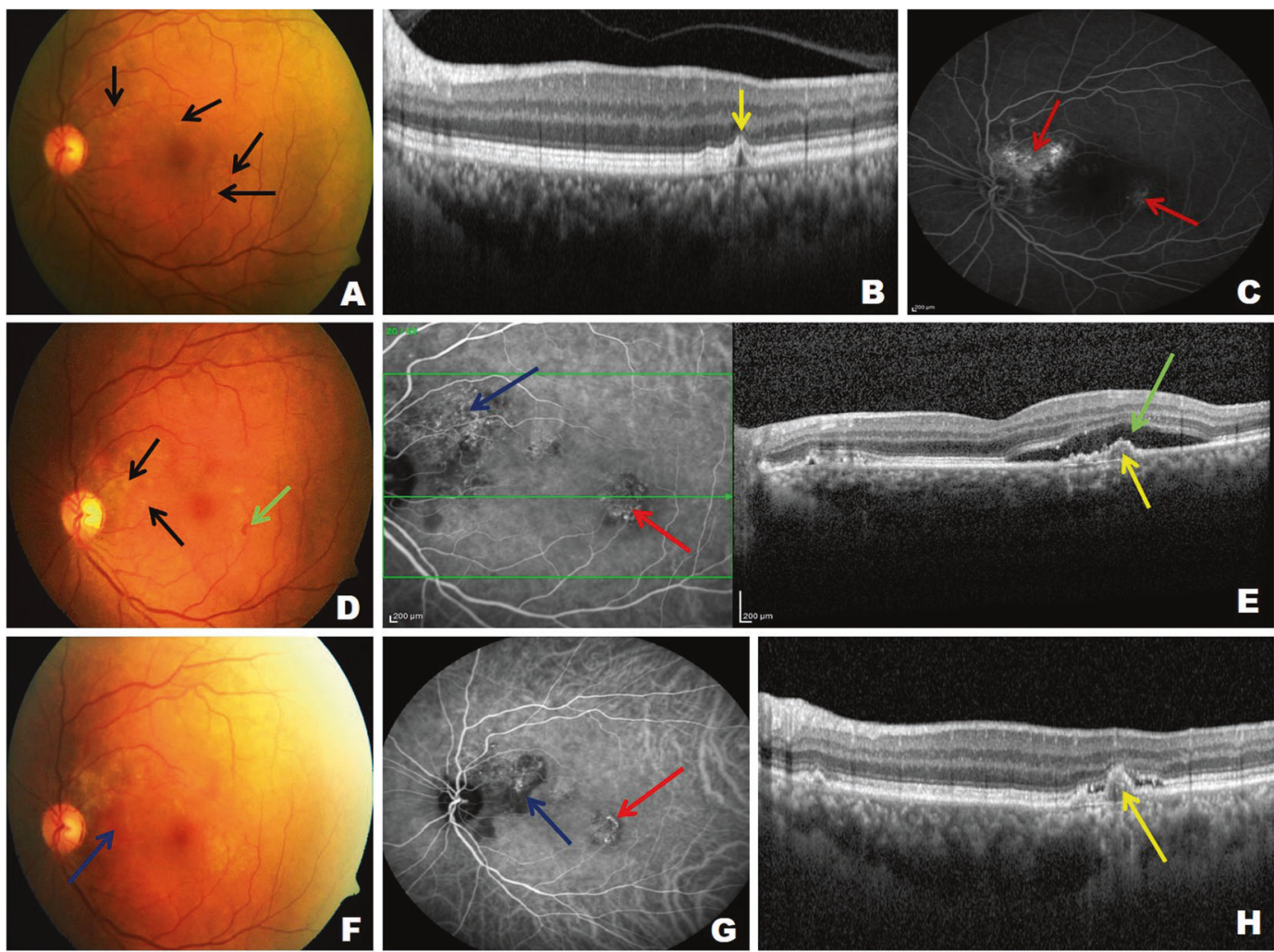

Fig. 1 Representative case of long term progression of pachydrusen. Serial follow-up images of a $52 \mathrm{Y} / \mathrm{F}$ who presented with pachydrusen (PD) at baseline on color fundus photo (CFP; black arrows; a) which appears as RPE irregularities on SD-OCT (yellow arrow; b): One year later, the patient presented with small hemorrhage inferotemporal to macula at the site of PD (Green arrow; $\mathbf{d}$ ) with appearance of new PD in peripapillary location (Black arrow; d). Eye-tracked ICGA + OCT showed shallow-irregular pigment epithelial detachment (SI-PED; yellow arrow; e) with serous macular detachment (SMD; green arrow; e) at the site of hemorrhage with the simultaneous ICGA showing corresponding branching vascular network (BVN; Red arrow; d) with an additional network in peripapillary location (Blue arrow; d). Digital fluorescein angiography showed features of occult CNVM corresponding to these two networks (red arrow; c). The patient underwent five doses of intravitreal ranibizumab over 9 months. Subsequently, the lesion inferotemporal to macula showed significant reduction in SMD, but there was appearance of small irregular PED on SD-OCT (yellow arrow; $\mathbf{h}$ ) which corresponded to polyps on ICGA (red arrow; g). The patient also developed peripapillary shallow hemorrhagic PED (blue arrow; f) which corresponded to the site of new PD which appeared at one year (black arrow; d). ICGA in corresponding location showed polyp with BVN with blocked fluorescence due to hemorrhagic PED. 
RPE and underlying Bruch's membrane. As noted in literature, such changes in AMD trigger the development of either CNVM or GA [7-9]. Based on this analogy, and with similar micromorphic damage to RPE and Bruch's membrane by drusens, PD can be a potential risk factor for the development of disease activity in pachychoroid disorder, namely CSCR leak and / or CNVM formation. This premise conceivably explains the development of disease activation at the site of PD in the current study, i.e., leak in eyes with PPE and CSCR and development of polyps / BVN in PCV eyes.

Our study has a few limitations. First, it is primarily a retrospective review study of multimodal imaging. In addition, we did not assess the choroidal morphology underlying the PD on OCT. Additionally, a minimum follow-up of one year may be short to evaluate progressive changes in pachydrusen. Also, it was a single grader evaluation of the images including choroidal thickness. The strengths of our study include an adequate sample size and epidemiological evaluation of a recent entity of PD in the spectrum of pachychoroid. By extrapolating the results of our study, we conceptualize that serial follow-up of patients with PD on OCT may provide us with better insight into the early recognition of disease activity, especially type 1 CNVM (PNV / PCV).

\section{Compliance with ethical standards}

Conflict of interest AS: CONSULTANT: for Novartis, Allergan, Bayer and Intas. FB: CONSULTANT: Allergan, Bayer, BoehringerIngelheim, Fidia Sooft, Hofmann La Roche, Novartis, NTC Pharma, Sifi, Thrombogenics, Zeiss. BDK: CLINICAL RESEARCH: Alcon, Alimera, Allegro, Allergan, Apellis, Clearside, Genentech, GSK, Ionis, jCyte, Novartis, Regeneron, ThromboGenics; CONSULTANT:
Alimera, Allegro, Allergan, Cell Care, Dose, Eyedaptic, Galimedix, Genentech, Glaukos, Interface Biologics, jCyte, Novartis, Ophthotech, Regeneron, Revana, Theravance Biopharma. NK: None. NP: None

Publisher's note Springer Nature remains neutral with regard to jurisdictional claims in published maps and institutional affiliations.

\section{References}

1. Spaide RF. Disease expression in nonexudative age-related macular degeneration varies with choroidal thickness. Retina. 2018;38:708-16.

2. Lee J, Byeon SH. Prevalence and clinical characteristics of pachydrusen in polypoidal choroidal vasculopathy: multimodal image study. Retina. 2019;39:670-8.

3. Chew EY, Clemons TE, Agrón E, Sperduto RD, Sangiovanni JP, Davis MD. Age-Related Eye Disease Study Research Group et al. Ten-year follow-up of age-related macular degeneration in the age-related eye disease study: AREDS report no. 36. JAMA Ophthalmol. 2014;132:272-7.

4. Buch H, Nielsen NV, Vinding T, Jensen GB, Prause JU, la Cour M. 14-year incidence, progression, and visual morbidity of age-related maculopathy: the Copenhagen City Eye Study. Ophthalmology. 2005;112:787-98.

5. Cheung CMG, Lee WK, Koizumi H, Dansingani K, Lai TYY, Freund KB. Pachychoroid disease. Eye (Lond). 2019;33:14-33.

6. Warrow DJ, Hoang QV, Freund KB. Pachychoroid pigment epitheliopathy. Retina. 2013;33:1659-72.

7. Williams MA, Craig D, Passmore P, Silvestri G. Retinal drusen: harbingers of age, safe havens for trouble. Age Ageing 2009;38: 648-54.

8. Nozaki M, Raisler BJ, Sakurai E, Sarma JV, Barnum SR, Lambris JD, et al. Drusen complement components C3a and C5a promote choroidal neovascularization. Proc Natl Acad Sci USA. 2006;103: 2328-33.

9. Boyer DS, Schmidt-Erfurth U, van Lookeren Campagne M, Henry EC, Brittain C. The pathophysiology of geographic atrophy secondary to age-related macular degeneration and the complement pathway as a therapeutic target. Retina. 2017;37:819-35. 